474/,/,/,A.G.Ramm, Discrepancy principle for the dynamical systems method, Communic. in Nonlinear Sci. and Numer. Simulation, 10, N1, (2005), 95-101 


\title{
Discrepancy principle for the dynamical systems method
}

Key words: ill-posed problems, dynamical systems method (DSM), discrepancy principle, evolution equations.

Math subject classification: 34R30, 35R25, 35R30, 37C35, 37L05, 37N30, 47A52, 47J06, 65M30, 65N21;

PACS 02.30.-f, 02.30.Tb, 02.30.Zz,02.60Lj, 02.60.Nm, 02.70.Pt, 05.45.-a

\author{
A.G. Ramm \\ Mathematics Department, Kansas State University, \\ Manhattan, KS 66506-2602, USA \\ ramm@math.ksu.edu
}

Assume that

Abstract

$A u=f$

is a solvable linear equation in a Hilbert space, $\|A\|<\infty$, and $R(A)$ is not closed, so problem (1) is ill-posed. Here $R(A)$ is the range of the linear operator $A$. A DSM (dynamical systems method) for solving (1), consists of solving the following Cauchy problem:

$$
\dot{u}=-u+(B+\epsilon(t))^{-1} A^{*} f, \quad u(0)=u_{0},
$$

where $B:=A^{*} A, \dot{u}:=\frac{d u}{d t}, u_{0}$ is arbitrary, and $\epsilon(t)>0$ is a continuously differentiable function, monotonically decaying to zero as $t \rightarrow \infty$. A.G.Ramm has proved that, for any $u_{0}$, problem (2) has a unique solution for all $t>0$, there exists $y:=w(\infty):=$ $\lim _{t \rightarrow \infty} u(t), A y=f$, and $y$ is the unique minimal-norm solution to (1). If $f_{\delta}$ is given, such that $\left\|f-f_{\delta}\right\| \leq \delta$, then $u_{\delta}(t)$ is defined as the solution to (2) with $f$ replaced by $f_{\delta}$. The stopping time is defined as a number $t_{\delta}$ such that $\lim _{\delta \rightarrow 0}\left\|u_{\delta}\left(t_{\delta}\right)-y\right\|=0$, and $\lim _{\delta \rightarrow 0} t_{\delta}=\infty$. A discrepancy principle is proposed and proved in this paper. This principle yields $t_{\delta}$ as the unique solution to the equation:

$$
\left\|A(B+\epsilon(t))^{-1} A^{*} f_{\delta}-f_{\delta}\right\|=\delta
$$

where it is assumed that $\left\|f_{\delta}\right\|>\delta$ and $f_{\delta} \perp N\left(A^{*}\right)$. For nonlinear monotone $A$ a discrepancy principle is formulated and justified. 


\section{Introduction and statement of the result.}

Assume that

$$
A u=f,
$$

is a solvable linear equation in a Hilbert space, $\|A\|<\infty$, and $R(A)$ is not closed, so problem (1.1) is ill-posed. Here $R(A)$ is the range of the linear operator $A$. Without loss of generality, assume that $\|A\| \leq 1$. Let $y$ be the unique minimal-norm solution to (1.1). A solvable quation (1.1) is equivalent to

$$
B u=A^{*} f, \quad B:=A^{*} A,
$$

where $A^{*}$ is the operator adjoint to $A$. One has $N(B)=N(A):=N:=\{v: A v=0\}$. Let $Q:=A A^{*}$, and $a>0$ be a number. Then $\|B\| \leq 1,\|Q\| \leq 1$, and $(B+a)^{-1} A^{*}=$ $A^{*}(Q+a)^{-1}$, as one easily checks. Denote by $E_{\lambda}$ and $F_{\lambda}$ the resolutions of the identity of $B$ and $Q$, respectively.

Let $\epsilon(t)$ be a monotone, decreasing function,

$$
\epsilon(t)>0, \quad \lim _{t \rightarrow 0} \epsilon(t)=0, \quad \lim _{t \rightarrow \infty} \sup _{\frac{t}{2} \leq s \leq t}|\dot{\epsilon}(s)| \epsilon^{-2}(t)=0
$$

A DSM (dynamical systems method) for solving (1.1), consists of solving the following Cauchy problem:

$$
\dot{u}=-u+(B+\epsilon(t))^{-1} A^{*} f, \quad u(0)=u_{0}, \quad \dot{u}:=\frac{d u}{d t}
$$

where $u_{0}$ is arbitrary, and proving that, for any $u_{0}$, problem (1.3) has a unique solution for all $t>0$, there exists $y:=u(\infty):=\lim _{t \rightarrow \infty} u(t), A y=f$, and $y$ is the unique minimal-norm solution to (1.1). These results are proved in [3]. If $f_{\delta}$ is given, such that $\left\|f-f_{\delta}\right\| \leq \delta$, then $u_{\delta}(t)$ is defined as the solution to (1.3) with $f$ replaced by $f_{\delta}$. The stopping time is defined as a number $t_{\delta}$ such that $\lim _{\delta \rightarrow 0}\left\|u_{\delta}\left(t_{\delta}\right)-y\right\|=0$, and $\lim _{\delta \rightarrow 0} t_{\delta}=\infty$. A discrepancy principle for choosing $t_{\delta}$ is proposed and proved in this paper.

Let us assume $f_{\delta} \perp N\left(A^{*}\right)$. Then this discrepancy principle, yields $t_{\delta}$ as the unique solution to the equation:

$$
\left\|A(B+\epsilon(t))^{-1} A^{*} f_{\delta}-f_{\delta}\right\|=\delta, \quad\left\|f_{\delta}\right\|>\delta,
$$

and we prove that

$$
\lim _{\delta \rightarrow 0}\left\|u_{\delta}\left(t_{\delta}\right)-y\right\|=0, \quad \lim _{\delta \rightarrow 0} t_{\delta}=\infty .
$$

Thus, our basic result is:

Theorem 1.1. If $A$ is a bounded linear operator in a Hilbert space $H$, equation (1.1) is solvable, $\left\|f_{\delta}\right\|>\delta, f_{\delta} \perp N\left(A^{*}\right)$, and $\epsilon(t)$ satisfies the assumptions stated above, then 
equation (1.4) has a unique solution $t_{\delta}$, and (1.5) holds, where $y$ is the unique minimalnorm solution to (1.1).

In Section 2 a proof of this theorem is given. For variational regularization the discrepancy principle was proposed by Morozov [2], see also [1] and [4]. In [5] a general regularization method is proposed for a wide class of nonlinear ill-posed problems.

\section{Proofs.}

First, let us prove two lemmas. By $\rightarrow$ and $\rightarrow$ we denote the weak and strong convergence in $H$, respectively.

Lemma 1. If $w_{n} \rightarrow y$, and $\limsup _{n \rightarrow \infty}\left\|w_{n}\right\| \leq\|y\|$, then $w_{n} \rightarrow y$.

Lemma 2. If $\epsilon(t)>0, \dot{\epsilon}<0$, and $\lim _{t \rightarrow \infty} \dot{\epsilon}(t) \epsilon^{-2}(t)=0$, then $\lim _{t \rightarrow \infty} e^{-t} \epsilon^{-1}(t)=0$.

Proof of Lemma 1. If $w_{n} \rightarrow y$, then $\|y\| \leq \liminf _{n \rightarrow \infty}\left\|w_{n}\right\|$. This, and the inequality $\lim \sup _{n \rightarrow \infty}\left\|w_{n}\right\| \leq\|y\|$ yield $\lim _{n \rightarrow \infty}\left\|w_{n}\right\|=\|y\|$. Thus, $\lim _{n \rightarrow \infty}\left\|w_{n}-y\right\|^{2}=$ $\lim _{n \rightarrow \infty}\left[\left\|w_{n}\right\|^{2}+\|y\|^{2}-2 \Re\left(w_{n}, y\right)\right]=0$.

Proof of Lemma 2. Our assumptions imply $\frac{d \epsilon^{-1}(t)}{d t} \leq c$, where $c=$ const $>0$. Thus $\epsilon^{-1}(t) \leq c t+c_{0}$, where $c_{0}>0$ is a constant. The conclusion of Lemma 2 follows.

Remark 1. An example of $\epsilon(t)$, satisfying all the assumptions imposed in the Introduction, is $\epsilon(t)=c_{1}\left(c_{0}+t\right)^{-b}$, where $c_{0}, c_{1}>0$ are positive constants, and $b \in(0,1)$ is a constant.

The proof of Theorem 1.1 consists of two steps.

Step 1: prove that (1.4) has a unique solution $t_{\delta}$, and $\lim _{\delta \rightarrow 0} t_{\delta}=\infty$.

Step 2: prove (1.5).

Step 1. Write $\epsilon$ for $\epsilon(t)$ and rewrite (1.4) as:

$$
\begin{gathered}
\delta^{2}=\left\|A(B+\epsilon)^{-1} A^{*} f_{\delta}-f_{\delta}\right\|^{2}=\left\|\left[Q(Q+\epsilon)^{-1}-I\right] f_{\delta}\right\|^{2}= \\
=\epsilon^{2} \int_{0}^{1}(\epsilon+\lambda)^{-2} d \rho(\lambda):=h(\delta, \epsilon) .
\end{gathered}
$$

Here $Q=A A^{*}, \rho=\left(F_{\lambda} f_{\delta}, f_{\delta}\right),\|Q\| \leq 1$. One has $h(\infty, \delta)=\int_{0}^{1} d \rho=\left\|f_{\delta}\right\|^{2}>\delta^{2}$, and $\lim _{\epsilon \rightarrow 0} h(\epsilon, \delta)=0$, by the dominant convergence theorem, provided that $\lim _{\gamma \rightarrow 0} \int_{0}^{\gamma} d \rho=0$. The last relation holds if $f_{\delta} \perp N\left(A^{*}\right)=N(Q)$. This assumption is natural, because $f_{\delta}$ enters under the sign of $A^{*}$ in the definition of $w_{\delta}(s)$ in the argument given in Step 2 . Thus, $h(\delta, \infty)>\delta^{2}$ and $h(\delta, 0)=0<\delta^{2}$. By the continuity of $h$ as a function of $\epsilon$ one concludes that there exists a solution $\epsilon:=g(\delta)>0$ to the equation $h(\delta, \epsilon)=\delta^{2}$. Since $h$ is a monotone increasing function of $\epsilon$, this solution is unique. Because $\epsilon(t)$ is monotone decreasing, the equation $\epsilon(t)=g(\delta)$ defines a unique root $t_{\delta}$, and $\lim _{\delta \rightarrow 0} t_{\delta}=\infty$. Step 1 is done.

Step 2. To prove (1.5), write $u_{\delta}(t)=u_{0} e^{-t}+\int_{0}^{t} e^{-(t-s)} w_{\delta}(s) d s$, where here and below $t:=t_{\delta}$ is defined in Step 1 , and $w_{\delta}(s):=(B+\epsilon(s))^{-1} A^{*} f_{\delta}$. Because $f_{\delta}$ enters under the sign of $A^{*}$, one may assume that $f_{\delta} \perp N\left(A^{*}\right)=N(Q)$. This assumption has been used in Step 1. 
We have $u_{\delta}=j_{1}+j$, where $j_{1}:=u_{0} e^{-t}+\int_{0}^{t / 2} e^{-(t-s)} w_{\delta}(s) d s$, and $j:=\int_{t / 2}^{t} e^{-(t-s)} w_{\delta}(s) d s$. One has $\left\|w_{\delta}(s) \mid \leq\right\| f_{\delta} \| \epsilon^{-1}(s)$, because $\left\|A^{*}\right\| \leq 1$, and $\left\|(B+\epsilon)^{-1}\right\| \leq \epsilon^{-1}$. By Lemma 2 ,

$$
\left\|j_{1}\right\| \leq\left\|u_{0}\right\| e^{-t}+e^{-t / 2} \epsilon^{-1}(t / 2)\left\|f_{\delta}\right\| \rightarrow 0 \quad \text { as } \quad t \rightarrow \infty .
$$

Furthermore,

$$
\|j-y\| \leq\left\|w_{\delta}(t) \int_{t / 2}^{t} e^{-(t-s)} d s-y\right\|+\left\|\int_{t / 2}^{t} e^{-(t-s)}\left[w_{\delta}(s)-w_{\delta}(t)\right] d s\right\|:=J_{1}+J_{2} .
$$

One has:

$$
J_{1}=\left\|w_{\delta}(t)-y-w_{\delta}(t) e^{-t / 2}\right\| \rightarrow 0 \quad \text { as } \quad t \rightarrow \infty,
$$

because, as we prove below,

$$
\lim _{\delta \rightarrow 0}\left\|w_{\delta}\left(t_{\delta}\right)-y\right\|=0
$$

To prove that $J_{2} \rightarrow 0$ as $t \rightarrow \infty$, we estimate

$\left\|w_{\delta}(s)-w_{\delta}(t)\right\|=\left\|(B+\epsilon(s))^{-1}(\epsilon(s)-\epsilon(t))(B+\epsilon(t))^{-1} A^{*} f_{\delta}\right\| \leq\left\|f_{\delta}\right\| \epsilon^{-2}(t)|\dot{\epsilon}(\xi)|(t-s)$,

where $t / 2 \leq s \leq t$, and $\xi$ is an intermediate point in the Lagrange formula. Thus,

$$
J_{2} \leq\left\|f_{\delta}\right\| \int_{t / 2}^{t} e^{-(t-s)}(t-s) d s \epsilon^{-2}(t) \sup _{\frac{t}{2} \leq \xi \leq t}|\dot{\epsilon}(\xi)| \rightarrow 0 \quad \text { as } \quad t \rightarrow \infty .
$$

Let us prove $(2.2)$. The element $w_{\delta}:=w_{\delta}\left(t_{\delta}\right)$ is the minimizer of the problem: $\left\|A w-f_{\delta}\right\|^{2}+\epsilon\|w\|^{2}=\min , \epsilon:=\epsilon(t), t:=t_{\delta}$. Thus,

$$
\left\|A w_{\delta}-f_{\delta}\right\|^{2}+\epsilon\left\|w_{\delta}\right\|^{2} \leq\left\|A(y)-f_{\delta}\right\|^{2}+\epsilon\|y\|^{2}=\delta^{2}+\epsilon\|y\|^{2} .
$$

So, $\left\|w_{\delta}\right\| \leq\|y\|$, because of (1.4). Therefore, one may assume that $w_{\delta} \rightarrow W$ as $\delta \rightarrow 0$, and, as we prove below, $W=y$. Now, Lemma 1 implies that (2.2) holds.

Let us prove that $W=y$. It follows from (1.4) that $B w_{\delta} \rightarrow A^{*} f$ as $\delta \rightarrow 0$. This and $w_{\delta} \rightarrow W$, imply $B W=A^{*} f$, since $B$ is monotone and therefore $w$-closed. We prove $w$-closedness of $B$ below. Since the minimal-norm solution to (1.2) is unique, and since $\|W\| \leq\|y\|$, it follows that $W=y$, as claimed.

Finally, we prove $w$-closedness of a monotone, hemicontinuous operator $B$ defined on all of $H$. We call an operator $B w$-closed if $w_{j} \rightarrow y$ and $B w_{j} \rightarrow f$ imply $B y=f$. An operator $B$ is called monotone, if $(B(u)-B(v), u-v) \geq 0$ for all $u, v \in D(B)$, and hemicontinuous, if the function $t \mapsto(B(u+t v), z)$ is continuous for any $u, v$, and $z \in H$, as a function of $t \in[0,1)$. To prove $w$-closedness of $B$, we start with the relation: $\left(B\left(u_{j}\right)-B(y-t z), u_{j}-y+t z\right) \geq 0$. Let $j \rightarrow \infty$. Then $(f-B(y-t z), t z) \geq 0$, or, since $t>0,(f-B(y-t z), z) \geq 0$. Let $t \rightarrow 0$. Then, by hemicontinuity of $B$, one gets $(f-B(y), z) \geq 0$. Since $z$ is arbitrary, it follows that $B y=f$, as claimed. Note, that if $B$ is continuous, it is hemicontinuous. Step 2 is done. Theorem 1.1 is proved. 
Remark 2. Suppose that the assumption $f_{\delta} \perp N\left(A^{*}\right)$ does not hold. Let $f_{\delta}=g+h$, where $g \perp h, g \in N\left(A^{*}\right)$. Then one can use the discrepancy principle of the form:

$$
\left\|A(B+\epsilon(t))^{-1} A^{*} f_{\delta}-f_{\delta}\right\|=C \delta, \quad\left\|f_{\delta}\right\|>C \delta, \quad C=\text { const }>1 .
$$

In Step 1 we prove now that $h(\delta, 0)<C^{2} \delta^{2}, h(\delta, \infty)>C^{2} \delta^{2}$, and the arguments are similar to the given in the proof of Theorem 1.1.

Remark 3. In this remark we sketch a proof of a discrepancy principle for nonlinear, monotone, continuous operators, defined on all of $H$. A related result for linear operators is proved in [6].

Theorem 2.1. Assume: i) A is a monotone, continuous, defined on all of $H$, operator, ii) equation $A(u)=f$ is solvable, $y$ is its minimal-norm solution, and iii) $\left\|f_{\delta}-f\right\| \leq \delta,\left\|A(0)-f_{\delta}\right\|>C \delta$, where $C>1$ is a constant which can be chosen arbitrarily close to 1 . Define $\epsilon(\delta)>0$ to be the minimal solution to the equation

$$
\left\|A\left(u_{\delta, \epsilon}\right)-f_{\delta}\right\|=C \delta
$$

where $u_{\delta, \epsilon}$ is any element satisfying inequality

$$
F\left(u_{\delta, \epsilon}\right) \leq m+\left(C^{2}-1\right) \delta^{2}, \quad F(u):=\left\|A(u)-f_{\delta}\right\|^{2}+\epsilon\|u\|^{2},
$$

and $m=m(\delta, \epsilon):=i n f_{u} F(u)$. Then equation (2.3) for $\epsilon$ has a solution $\epsilon(\delta)>0$. If $\epsilon(\delta)$ is its minimal solution, and $u_{\delta}:=u_{\delta, \epsilon(\delta)}$, then $\lim _{\delta \rightarrow 0}\left\|u_{\delta}-y\right\|=0$.

Sketch of Proof. If $A$ is monotone, continuous, defined on all of $H$, then the set $N_{f}:=\{u: A(u)=f\}$ is convex and closed, so it has a unique minimal-norm element $y$. To prove the existence of a solution to (2.3), we prove that the function

$$
h(\delta, \epsilon):=\left\|A\left(u_{\delta, \epsilon}\right)-f_{\delta}\right\|
$$

is greater than $C \delta$ for sufficiently large $\epsilon$, and smaller than $C_{1} \delta$ for sufficiently small $\epsilon$ and $C_{1}>C$ arbitrarily close to $C$. Because of the continuity of $h(\delta, \epsilon)$ with respect to $\epsilon$ on $(0, \infty)$, equation $h(\delta, \epsilon)=C \delta$ has a solution if $C>1$ and $C \delta<\left\|A(0)-f_{\delta}\right\|$.

Let us prove the above estimates. One has

$$
\epsilon\left\|u_{\delta, \epsilon}\right\|^{2} \leq F\left(u_{\delta, \epsilon}\right) \leq m+\left(C^{2}-1\right) \delta^{2} \leq F(0)+\left(C^{2}-1\right) \delta^{2}
$$

and

$$
h^{2}(\delta, \epsilon) \leq F\left(u_{\delta, \epsilon}\right) \leq m+\left(C^{2}-1\right) \delta^{2} \leq F(y)+\left(C^{2}-1\right) \delta^{2}=\epsilon\|y\|^{2}+C^{2} \delta^{2} .
$$

Therefore, as $\epsilon \rightarrow \infty$, one gets $\left\|u_{\delta, \epsilon}\right\| \leq \frac{c}{\sqrt{\epsilon}} \rightarrow 0$, where $c>0$ is a constant depending on $\delta$. Thus, by the continuity of $A$, one obtains $\lim _{\epsilon \rightarrow \infty} h(\delta, \epsilon)=\left\|A(0)-f_{\delta}\right\|>C \delta$.

Now, let $\epsilon \rightarrow 0$. Then $h^{2}(\delta, \epsilon) \leq \epsilon\|y\|^{2}+C^{2} \delta^{2}$. Thus $\liminf _{\epsilon \rightarrow 0} h(\delta, \epsilon) \leq C \delta$. Therefore equation $h(\delta, \epsilon)=C_{1} \delta$ has a solution $\epsilon(\delta)>0$ if $C<C_{1}<\left\|A(0)-f_{\delta}\right\|$, which is what we 
want, because $C_{1}>1$ can be taken arbitrarily close to 1 if $C>1$ can be taken arbitrarily close to 1 .

Let us now prove that if $u_{\delta}:=u_{\delta, \epsilon(\delta)}$, then $\lim _{\delta \rightarrow 0}\left\|u_{\delta}-y\right\|=0$. From the estimate

$$
\left\|A\left(u_{\delta}\right)-f_{\delta}\right\|^{2}+\epsilon\left\|u_{\delta}\right\|^{2} \leq C^{2} \delta^{2}+\epsilon\|y\|^{2}
$$

and from the equation (2.3), it follows that $\left\|u_{\delta}\right\| \leq\|y\|$. Thus, one may assume that $u_{\delta} \rightarrow U$, and from (2.3) it follows that $A\left(u_{\delta}\right) \rightarrow f$ as $\delta \rightarrow 0$. By $w$-closedness of monotone continuous operators, one gets $A(U)=f$, and from $\left\|u_{\delta}\right\| \leq\|y\|$ it follows that $\|U\| \leq\|y\|$. Because the minimal norm solution to the equation $A(u)=f$ is unique, one gets $U=y$. Thus, $u_{\delta} \rightarrow y$, and $\left\|u_{\delta}\right\| \leq\|y\|$. By Lemma 1, it follows that $\lim _{\delta \rightarrow 0}\left\|u_{\delta}-y\right\|=0$. Theorem 2.1 is proved.

\section{References}

[1] Engl H., Hanke M., Neubauer A., Regularization of Inverse Problems, Kluwer, Dordrecht, 1996.

[2] Morozov V., Methods of Solving Incorrectly Posed Problems, Springer Verlag, New York, 1984.

[3] Ramm, A.G., Dynamical systems method for solving operator equations, Communic. in Nonlinear Sci. and Numer. Simulation, 9, N2, (2004), 383-402

[4] Ramm, A.G., On the discrepancy principle, Nonlinear Functional Anal. and Applic., 8, N2, (2003), 307-312

[5] Ramm, A.G., Regularization of ill-posed problems with unbounded operators, J. Math. Anal. Appl., 271, (2002), 547-550.

[6] Ramm, A.G., A new discrepancy principle, J. Math. Anal. Appl., 310, (2005), 342345. 